\title{
VIRTUAL TEAM WORKING: CURRENT ISSUES AND DIRECTIONS FOR THE FUTURE
}

\author{
Aisha Abuelmaatti and Yacine Rezgui \\ Built and Human Environment Research Institute, University of Salford, Salford, UK. \\ a.m.t.abuelmaatti@pgr.salford.ac.uk and y.rezgui@salford.ac.uk
}

\begin{abstract}
Value networks underpinned by global and localised virtual teams are believed to have high potential for SMEs. Yet, the successful migration to value-added alliances is blended in the right combination of organizational, legal, economic, socio-cultural, and technical factors, and requires further research into innovative business models. These models should leverage existing SME competences and transcend existing virtual collaboration barriers and limitations. Grounded in state-of-the-art literature, the paper identifies current insight for the deficient research in virtual teams and presents them in the form of open research questions.
\end{abstract}

\section{INTRODUCTION}

Virtual team working is a concept that has matured through a long evolutionary process (Maznevski and Chudoba, 2000; Carmarinha-Matos and Afsarmanesh, 2005). While organisations emerge and claim to have adopted the modus operandi of virtual teams, the reality is that conventional face-to-face modes of operation will remain the organisational norm for some time to come (Arnison and Miller, 2002). Yet, the rapid pace of globalisation, the increasing need for agility, and the fast development of Information and Communication Technologies (ICT) will force organisations to embrace virtual collaboration to enhance their competitiveness (Arnison and Miller, 2002; Rezgui, 2007; Workman, 2007).

Small and Medium Sized Enterprises (SMEs) form a large proportion of organizations in Europe. While large enterprises have the advantage of taking on mass tasks, the size of SMEs tied to their limited resources prevent them from sustaining their competitiveness with larger organizations. However, SMEs exhibit advantages compared to large enterprises, in particular when it comes to adapting to changes and adopting technology (Rezgui, 2007).

Sustainable competitive advantage is interwoven with innovation (Barrett and Sexton, 2006). In this context, value-added alliance formation can be seen as an innovation and is essential in the current dynamic business environment (Helling et al., 2005). While a number of requirements emerge to support the migration of SMEs from traditional organizations to empowered alliances, a number of barriers hinder this migration. These include factors related to culture, organizational structure, decision making processes, perceptions in relation to change, shared 
responsibility management, liability, copyright and confidentiality issues, trust, employee-manager relationships, management strategies, and ICT maturity and capability (Rezgui 2007).

Barrett and Sexton (2006) define the process of innovation as a cyclical process of diagnosing, action planning, taking action, evaluating and specifying learning. It is vital to note that when operating in global markets, the criteria for competitiveness change continuously. A value network or alliance cycle starts with sensing an opportunity or need to innovate in response to competitive conditions. In particular, the SMEs' motivation to innovate is not solely to grow, but can be directed at competing with larger enterprises. A Value network underpinned by virtual team working is not an end in itself but can be a means to achieve sustainable competitiveness. In fact, SMEs consider virtual teams as (a) innovations with the potential to respond to complex business environments (Workman and Kahnweiler, 2001); (b) provide purported benefits (Rezgui and Wilson, 2005), and (c) create opportunities that are not found in traditional teams (Barrett and Sexton, 2006). Despite the rapid growth of virtual team innovations, their performance is far below their potential. Although research has helped to speculate success, global SME virtual teams face significant organisational, economic, legal, socio-cultural and technical challenges (Kayworth and Leidner, 2000; Zigurs, 2003). It seems easy for academics to research and develop virtual team solutions, yet the reality of deployment and adoption is complex given the numerous types of challenges faced by the virtual team.

In order to reach the optimal level of virtual team functioning, given the complexity of this phenomenon as well as the lack of research to date, further research into innovative business models that leverage existing SME competences and transcend current organisational, economic, legal, socio-cultural and technical barriers and limitations is therefore an important prerequisite. The paper identifies a gap in formal theories, structure, modelling, and life cycle behaviour of virtual teams and alliances. Grounded in state-of-the-art literature, the paper identifies current barriers, limitations and insight for the deficient research in virtual teams and expresses these in the form of open research questions. Moreover, virtual team working challenges are reviewed by integrating recent literature in response to the growing awareness of the need for formal business models for SMEs. On the basis of this current literature review, a proposition for future direction is presented.

\section{METHODOLOGICAL CONCEPTUAL FRAMEWORK}

A comprehensive literature review has been carried out targeting virtual team research. The conceptual framework underpinning the review is illustrated in Figure 1 , while the key references organized by conceptual area are given in Table 1. 


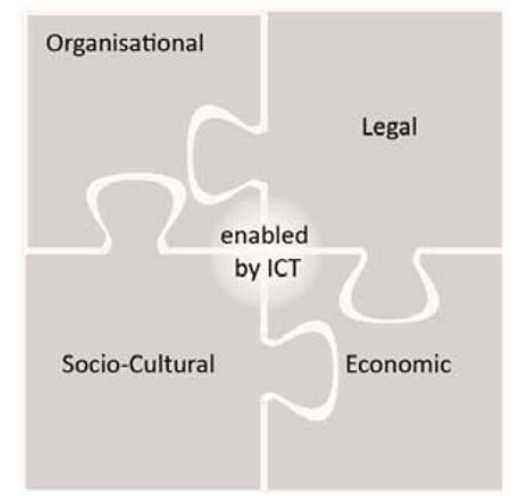

Figure 1 - Research Conceptual Framework.

Table 1 - Key references

\begin{tabular}{|l|l|}
\hline Organisational & Socio-cultural \\
Zigurs, 2003 & Zigurs, 2003 \\
Rezguiüoglu et al., 2005 & Kayworth and Leidner, 2000 \\
Kaiser et al., 2000 & McDonough et al., 2000 \\
Kaywoth and Leidner, 2000 & Arnison and Miller, 2002 \\
McDonough et al., 2000 & Workman et al., 2003 \\
Workman, 2001 & Kürümlüoglu et al., 2005 \\
Vakola and Wilson, 2004 & Rezgui and Wilson, 2005 \\
Rezgui, 2007 & Rezgui, 2007 \\
Pawar and Sharifi, 2000 & Wiesenfeld et al., 2000 \\
Barrett and Sexton, 2006 & Hoefling, 2001 \\
& Mezgár, 2006 \\
Legal & Arnison and Miller, 2002 \\
Shelbourn et al., 2005 & Pawar and Sharifi, 2000 \\
Economic & Yukl, 2002 \\
\hline Walker, 2000 & Connaughton and Daly, 2004 \\
Arnison and Miller, 2002 & Walters, 2000 \\
Lipnack and Stamps, 2000 & \\
Franke, 2001 & \\
Coulson and Kantamneni, 2006 & \\
Alsakini et al., 2007 & \\
Helling et al., 2005 & \\
\hline
\end{tabular}

\section{ORGANISATIONAL DIMENSION OF VIRTUAL TEAMS}

Virtual team working relies on the wide use of ICT, nevertheless handling the barriers and limitations of organizational structure, decision making, and perception in relation to change are fateful. This section addresses apiece respectively.

\subsection{Structure}

Collaboration gives rise to the fundamental requirements of labour division into tasks and the coordination of these tasks. The structure of an SME is reflected in the 
ways in which it divides its labour into distinct tasks and then achieves coordination among them. Virtual teams research to date has focused on the necessity of restructuring traditional organisational structures to exploit the fast development of ICTs (Zigurs, 2003; Kürümlüoglu et al., 2005; Rezgui and Wilson, 2005). In review of the substantial research on team structure in the traditional environment, coordination difficulties facing virtual teams have been found uncounted for. The literature relating to the structure of virtual working has put forward some suggestions attempting to achieve high team performance (Kaiser et al., 2000; Kaywoth and Leidner, 2000; McDonough et al., 2000; Workman, 2001). Yet, as managerial structures are associated with poor virtual SME alliance performance (Zigurs, 2003; Vakola and Wilson, 2004; Rezgui, 2007), the lack of structures handling virtual team working came under light. As such, the nature of the virtual SME alliance requires fresher approaches, thus providing fertile grounds for future research.

Further research should address: what structural work arrangements are best suited to the work that must transcend geographical boundaries and time? How SMEs effectively enforce these structures? What are the necessary abilities of the manager to facilitate communication among team members to create clear structures and foster role clarity to improve collaboration? Are there other strategies that SMEs can implement to improve virtual team working performance?

\subsection{Decision making and perception in relation to change}

SMEs find themselves in an almost constant state of change as they strive to respond to the pressure of the increasingly globalised and competitive environment. Thus, quick decision-making and innovation activity in response to rapidly changing conditions and demands is necessary (Pawar and Sharifi, 2000; Barrett and Sexton, 2006). The creation and operation of the SME alliance is regarded as a change initiative within the participating SMEs. Its members are likely to experience lifecycle problems- set up, operation, and winding down, where each of these different phases is likely to involve change in staffing, tasks, objectives and resources (Rezgui and Wilson, 2005). While most research in this area has been unable to break away from the traditional models, Rezgui and Wilson (2005) thoroughly reviewed the barriers and argued for a fresher approach. Future research in this area poses the questions of: what tasks enable perception, awareness, and preparedness to change? Do traditional managerial change mechanisms remain applicable in the virtual SME alliance environment? Either wise, what are the most appropriate change mechanisms? What business and organizational methods offer innovative and sustainable services along the collaboration? What formulas, depending on the nature and scale of the SME changes, are effective for decisionmaking? What is the necessary vision and systemic thinking required to manage the change lifecycle?

\section{LEGAL DIMENSION OF VIRTUAL TEAMS}

A typical process in the virtual team working is the removal or inclusion of participants. Virtual teams involve cooperation between legally independent SMEs. 
The fact that a virtual team has a legal identity does not mean that claims cannot be addressed directly towards the members. However, claimants will probably suffer some difficulties in determining the exact identity of the different members because of the appearance of the SME alliance as one enterprise (Shelbourn et al., 2005). To this end, attention should be paid to liability sharing and distribution.

SMEs use of "virtual teams" arises unanswered legal questions. The legal status still has to find a coherent framework and has not yet been adequately discussed. To ensure that SMEs are efficiently supported along their virtual collaboration path to delivering innovative solutions requires addressing the following issues: How to manage intellectual property rights and cope with copyright and confidentiality issues? How to manage responsibility? How to share and distribute liability? How to monitor these throughout collaboration? How shared responsibility by means of rights and ownership of outcomes is identified? How these foundations can be blended together to generate the basic building block to deliver sound legal entity?

\section{ECONOMIC DIMENSION OF VIRTUAL TEAMS}

The rapid pace of ICT has transformed the traditional economy into a smart new economy (Walker, 2000; Arnison and Miller, 2002). Pressures are forcing SMEs to become more adaptive and agile in their tasks and adopt innovative approaches. As a result, virtual teams have the potential to improve quality and performance and leverage capabilities (Lipnack and Stamps, 2000). Economic activity in this context means the cooperation of production ingredients to achieve competitiveness and maintain good cooperation between members of the SME alliance (Franke, 2001; Coulson and Kantamneni, 2006; Alsakini et al., 2007).

While a number of studies (Lipnack and Stamps, 2000; Walker, 2000; Franke, 2001; Arnison and Miller, 2002; Coulson and Kantamneni; 2006; Helling et al., 2005; Alsakini et al., 2007) discussed the collaborative networks' economic dimension, the complex business environment poses persistent problems to SMEs. From the economic standpoint, achieving competitiveness and maintaining good cooperation cannot depend solely on mutual faith. Research is needed to devise how to share profits and losses in the context of an SME alliance? How to ensure that the collective financial gain of the SME alliance outweighs the individual profits of associated member SMEs? How SMEs evaluate and determine the right economic costing in a consistent manner across the network?

\section{SOCIO-CULTURAL DIMENSION OF VIRTUAL TEAMS}

Socio-cultural barriers and limitations of maintaining virtual working teams are highlighted by integrating present literature on trust, social cohesion, team member structure - user / manager relationships, influences on the management and strategies.

The core of research arguments on trust centers on a belief that only trust can prevent the geographical boundaries and time zones of virtual team members from becoming psychological distances (Zigurs, 2003). Several suggestions to manipulate 
trust are present (Kayworth and Leidner, 2000; McDonough et al., 2000; Arnison and Miller, 2002; Workman et al., 2003; Kürümlüoglu et al., 2005; Rezgui and Wilson, 2005; Rezgui, 2007). Yet, such trust albeit swift is known to be fragile (Wiesenfeld et al., 2000; Hoefling, 2001; Zigurs, 2003; Mezgár, 2006).

Research suggests that face-to-face interaction has a direct impact on team performance through building team trust and enabling team members to exchange valuable socio-cultural information (Arnison and Miller, 2002; Rezgui, 2007). Research stresses the need for initial face-to-face meeting to provide the grounds for a worthwhile ICT collaboration (Kürümlüoglu et al., 2005). Extending this idea even further, research suggests that virtual team members conduct periodic face-to-face meetings (Kürümlüoglu et al., 2005; Rezgui, 2007).

It is essential that team managers play a pivotal role in favour of relationships (Kayworth and Leidner, 2000; McDonough et al., 2000; Pawar and Sharifi, 2000; Arnison and Miller, 2002; Yukl, 2002; Connaughton and Daly, 2004; Kürümlüoglu et al., 2005; Rezgui and Wilson, 2005). Relationship management ought to influence a strategy that identifies and maintains relationships which in turn ensures that objectives meet expectations (Walters, 2000). Rezgui (2007) accentuated this issue calling for a certain shift in the leadership approach identifying the need for essential attributes.

Seeing the decades of traditional team working, the legitimate question posing itself here is whether virtual teams can function effectively in the absence of frequent face-to-face communication? Further research should address what facts pave the way to foster swift trust? How is trust maintained? What working infrastructures utilized by teams attempt to foster trust? Which, if any, team training accustoms expert team members in their fields to the particular requirements of virtual working? What can relationship management do to foster teams of mixed experiences? How would members relate and identify themselves to their manager in a virtual context? What are the qualities that a virtual team manager ought to have to cope with the complexity resulting from non-collocation and virtual collaboration including trust, lack of cohesion and resolving issues? In the worst case scenario, what requirements the team needs to benefit from the diversity and dispersion regardless of trust?

The fact remains that the organisational culture is a critical factor to hold virtual teams. What remains unclear are how team members in a virtual context build, sustain and strengthen culture in the absence of frequent face-to-face interaction? How often should the team members communicate to remain glued? How to foster a culture of extensive collaboration? What behaviours inhibit a team's ability to develop a shared culture? What behaviours raise a team's ability to develop a shared culture? What current SMEs culture circumstances hinder team effectiveness in the virtual environment? Can a set of cultural attributes that promote effectiveness of teams be identified? How can these attributes be effectively enforced in virtual teams to ensure that members remain glued?

\section{TECHNOLOGICAL DIMENSION OF VIRTUAL TEAMS}

A technological solution in the context of virtual teams has to support the central 
business processes; allow integration of systems and interoperability between disparate applications; and the management of interactions between individuals and teams (Rezgui, 2007). A number of researchers have proposed to adopt approaches that federate services from various non-collocated organisations and software houses and making the applications they offer available via ubiquitous web browsers. This is commonly known as service composition.

As largely reported in the literature, web service composition is a very complex and challenging task. A number of key issues emerge from the literature as essential to support effectively service composition in favour of virtual team working, including: Coordination (to manage interaction between services and coordination of sequences of operations, to ensure correctness and consistency); Transaction (to manage short-duration / atomic and long running business activities); Context (to adjust execution and output to provide the client with a customised and personalised behaviour: may contain information such as a consumer's name, address, and current location, the type of client device, including hard- and software that the consumer is using, or all kinds of preferences regarding the communication); Conversation modelling (to facilitate service discovery and dynamic binding, service composition model validation, service composition skeleton generation, analysis of compositions and conversations and conversation model generation); Execution monitoring (involves either centralised or distributed execution of composite web services). On the other hand, existing web service engines are ill-suited to support the dynamic and changing nature of service environments. The paper argues that a number of key limitations emerge, which hinder full exploitation of web services as a promising middleware technology to support virtual team working, including:

- Existing service description and Web Service flow languages are ill suited when addressing the dynamics and non-functional characteristics of distributed business processes. The current Business Process Execution Language (BPEL) version does not support run-time alterations to address unforeseen problems, such as the replacement or addition of a new Web Service. In order to manage this uncertainty, BPEL processes need to have the ability to be extended to meet unforeseen post-deployment requirements and user needs.

- Web service flow engines, such as the ones implemented to support BPEL, lack execution monitoring functionality to manage the running process. These can help debug processes during development stage, with monitoring, and even be driven by agents at production stage. It is possible, for example, to embed, without modifying the engine implementation, a planner on the top of the latter. From events triggered by a monitor, this planner can take actions to avoid any disruption and to adjust the process. Such a tool can be useful particularly for long running processes.

- Web service composition methodologies have a focus on syntactic integration and therefore do not support automatic composition of web services. Semantic integration is crucial for web services as it allows them to (a) represent and reason about the task that a web service performs, (b) explicitly express and reason about business relations and rules, (c) understand the meaning of exchanged messages, (d) represent and reason about preconditions that are required to use the service and the effects of 
having invoked the service, and (e) allow intelligent composition of web services to achieve a more complex service.

Also, long running virtual team processes are subject to evolutions and change of different nature: process model evolution due to change in the environment (change in the law, change in the methodology), process instance evolution (or ad-hoc evolution) due to specific events occurring during a given process execution (delay, new available or lack of resources) or partnership evolution at execution time having an impact on part of the process. These shortcomings require essential advances and improvements.

The research suggests that new forms of software licensing are needed to provide a better software service that includes configuration, maintenance, training and access to a help-desk to ensure that SMEs are efficiently supported along their path to engage effectively in virtual teams.

\section{CONCLUSION}

Original motivation of the analysis in this paper was to review present virtual team working research. However, the lack of present research made additional research questions equal focus of the paper. The characteristics of SMEs suggest that in researching, developing, and evaluating potential virtual teamwork solutions, the human and organisational aspects require close attention. This means that social and, ultimately, economical considerations have to be made rather than concentrating the development process on the technology alone as has been traditionally the case.

Given current limitations of virtual team research, the paper contributes to existing knowledge by raising a number of research questions related to (a) clarifying and defining the nature of teamwork that takes place amongst SMEs, (b) specifying the technological, regulatory and socio-organisational environment to support team working effectively; and (c) researching into factors that facilitate virtual team adoption and use across SMEs and more generally in relation to any organization. Also, while existing research has provided little formalization of working procedures and managerial structures of virtual teams, the paper calls for further research in (a) technology maturity and software provision models, (b) organisational and process settings, and (c) social, including socio-emotional considerations, adapted to the needs of SMEs. It is hoped that the paper will trigger further research that will contribute to develop a holistic understanding of the complex theme of Virtual Teams.

\section{REFERENCES}

1. Alsakini W, Kiiras J, Huovinen P. "Competitive Virtuality among Construction Management Services Company". In Encyclopaedia of Networked and Virtual Organizations, Goran D. Putnik, Maria M. Cunha, eds. Information Science Reference, 2006

2. Arnison L, Miller P. Virtual teams: a virtue for the conventional team. Journal of Workplace Learning 2002; 14: 166-173. 
3. Barrett P, Sexton M. Innovation in small, project-based construction firms. British Journal of Management 2006; 17: 331-346.

4. Carmarinha-Matos LM, Afsarmanesh H. "Base concepts". In Virtual Organizations Systems and Practices, Luis M. Camarninha-Matos L, Hamideh Afsarmanesh, Martin Ollus, eds. New York: Springer Science, 2005.

5. Connaughton S, Daly J. Identification with leader: a comparison of perceptions of identification among geographically dispersed and co-located teams Corporate Communications: An International Journal 2004 9: 89-103.

6. Coulson and Kantamneni, Virtual corporations: the promise and perils. DC Press: 2006; www.dcpress.com/jmb/virtual.htm: last accessed 27/12/07

7. Franke, UJ. Virtual web organizations \& market conditions. The electronic journal of organizational virtualiness: 2001; 3: 43-64.

8. Helling K, Blim M, O'Regan B. An appraisal of virtual networks in the environmental sector. Management of Environmental Quality: An International Journal 2005; 16: 327 337.

9. Hoefling T. Working Virtually: Managing People for Successful Virtual Teams and Organisations. Stylus Publishing, 2001.

10. Kaywoth T, Leidner D. The global virtual manager: a prescription for success. European Management Journal 2000; 18: 183-194.

11. Kaiser P, Tullar W, McKowen D. Student team projects by internet. Business Communication Quarterly 2000; 63: 75-82.

12. Kürümlüoglu M, and Nøstdal R, Karvonen I: "Base concepts”. In In Virtual Organizations Systems and Practices, Luis M. Camarninha-Matos L, Hamideh Afsarmanesh, Martin Ollus, eds. New York: Springer Science, 2005.

13. Lipnack J, Stamps J. Virtual Teams: People Working Across Boundaries With Technology. New York: John Wiley \& Sons, 2000.

14. Maznevski ML, Chudoba KM. Bridging space over time: global virtual team dynamics and effectiveness. Organisation Science 2000; 11: 473-492.

15. McDonough EF, Kahn KB, Barczak G. An investigation of the use of global, virtual and collocated new product development teams. The Journal of Product Innovation Management 2000; 18: 110-120.

16. Mezgár I. Integration of ICT in Smart Organizations. Idea Group Inc, 2006.

17. OASIS, BPEL specification, http://www.oasis-open.org/committees/tc_home.php?wg abbrev=wsbpel last accessed 05/02/08.

18. Pawar KS, Sharifi S. Virtual collocation of design teams: coordinating for speed. International Journal of Agile Management Systems 2000; 2: 104-113.

19. Rezgui Y, Wilson I. "Socio-organizational issues". In Virtual Organizations Systems and Practices, Luis M. Camarninha-Matos L, Hamideh Afsarmanesh, Martin Ollus, eds. New York: Springer Science, 2005.

20. Rezgui Y. Exploring virtual team-working effectiveness in the construction sector. Interacting with Computers 2007; 19: 96-112.

21. Shelbourn M, Hassan T, Carter C. "Legal and contractual framework for the VO". In Virtual Organizations Systems and Practices, Luis M. Camarninha-Matos L, Hamideh Afsarmanesh, Martin Ollus, eds. New York: Springer Science, 2005.

22. Vakola M, Wilson IE. The challenge of virtual organization: critical success factors in dealing with constant change. Team Performance Management 2004; 10: 112-120.

23. Walker JW. E-leadership?. Human Resource Planning 2000; 23: 5-6.

24. Walters D. Virtual organizations: new lamps for old?. Management Decision 2000; 38: 420-436.

25. Wiesenfeld BM, Raghuram S, Garud R. Communication patterns as determinants of organizational identification in a virtual organization. Organization Science 2000; 10: 777-790. 
26. Workman M. Collectivism, individualism, and cohesion in a team-based occupation. Journal of Vocational Behavior 2001; 58: 82-97.

27. Workman M, Kahnweiler W, Bommer W. The effects of cognitive style and media richness on commitment to telework and virtual teams. Journal of Vocational Behaviour 2003; 63: 199-219.

28. Workman M. The effects from technology-mediated interaction and openness in virtual team performance measures. Behaviour and Information Technology 2007; 26: 355365.

29. Yukl G. Leadership in organizations. Englewood Cliffs, NJ: Prentice-Hall Inc, 2002.

30. Zigurs I. Leadership in virtual teams: oxymoron or opportunity?. Organizational Dynamics 2003; 31: 339-351. 